\title{
Gas Dynamic Simulations of Inner Regions of Protoplanetary Disks in Young Binary Stars
}

\author{
A. M. Fateeva*, D. V. Bisikalo, P. V. Kaygorodov, A. Y. Sytov \\ Institute of Astronomy of the Russian Acad. of Sci., 48 Pyatnitskaya, Moscow, Russia \\ * email: fateeva@inasan.ru
}

Keywords. protoplanetary disk, accretion, gap, binary stars, T Tauri stars

We have carried out 2D and 3D numerical simulations (Kaigorodov et al. 2010, Fateeva et al. 2011, Sytov et al. 2011) of accretion processes in binary T Tauri stars (TTSs) DQ Tau, UZ Tau E, V4046 Sgr, GW Ori, RoXs 42C using a finite-difference Roe-Osher-Einfeld TVD scheme. The morphology of the flow pattern for UZ Tau E is shown in Fig. 1 (left panel). The flow structure includes accretion disks surrounding the components, bow-shocks in front of both the components, a shock wave ("bridge") between the circumstellar accretion disks and a gap containing rarefied gas in the inner part of the protoplanetary disk.

The performed simulations show that the radii of the gaps which formed due to the bow-shocks fit the observations better than the radii calculated using positions of the Linblad resonances according to Artymowicz \& Lubow (1994). For systems with circular orbits, the calculated gap radius is $\sim 3 \mathrm{~A}$ ( $\mathrm{A}-$ semi-major axis of the system) and for those with elliptic orbits it is $\sim 3.2$ $-3.3 \mathrm{~A}$. Thus, the bow-shocks govern the size and shape of the gap in young binary systems.

Analysis of the fluxes demonstrates that the re-distribution of the angular momentum in the envelope due to the bow-shocks leads to occurrence of two flows propagating from the inner edge of the protoplanetary disk to the components (see Fig. 1, right panel). Let us consider streams near the less massive star (secondary). The matter in the gap splits into two streams at the head-on collision point when passing through the bow-shock. The first portion of matter (stream A in Fig. 1, right panel) loses its angular momentum at the shock and starts to move toward the circumstellar accretion disk forming a spiral flow. The second portion of matter
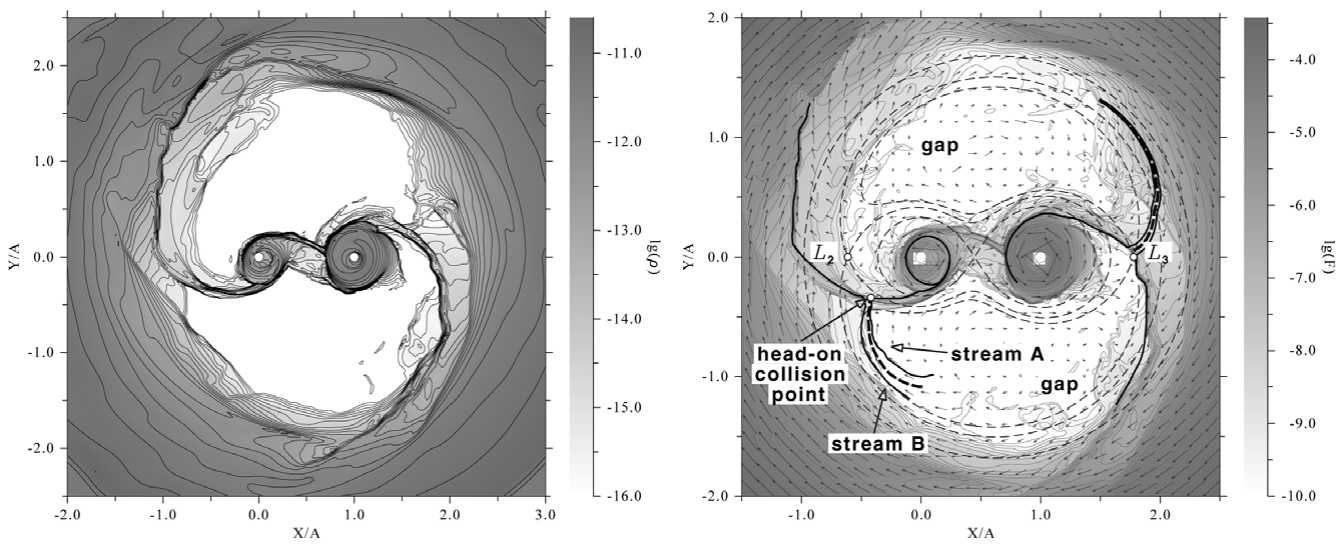

Figure 1. Results of 3D simulations for UZ Tau E. Left panel: the density distribution in the equatorial plane of the system is presented. Density isolines are also depicted. Right panel: the distribution of the matter flux and the velocity field in the equatorial plane of the system are presented. The flow lines corresponding to the main matter streams in the system are also shown by bold solid lines. The Roche equipotentials are depicted by the dashed line. 
(stream B) moves from the head-on collision point along the bow-shock to the protoplanetary disk carrying out the excess of the angular momentum. The gap size depends on the power of this flow. The same flow structure exists in the vicinity of the primary component.

The presented analysis of the streams allows us to make conclusions on the rates of accretion onto each component of the system. The secondary star moves faster than the primary one. It means that gas loses more angular momentum at the secondary's stronger shock and finally it leads to the higher flux toward the secondary. Furthermore, the secondary is located closer to the edge of the protoplanetary disk where gas is denser; hence, the flux onto this component must increase even more. Indeed, analysis of the fluxes demonstrates that, starting from the head-on collision point, the matter flux along the bow-shock of the secondary notably exceeds the same flux along the shock of the primary.

However, an accretion disk may not accept more matter than it is allowed by viscosity. The rest of the matter rounds the disk and collides with the same stream of another component. As a result, a bridge-like stationary shock between the circumstellar accretion disks of the components is formed (see Fig. 1, right panel). Due to this collision, the streams lose more angular momentum, since they are partially annihilated. The matter, having lost its angular momentum, is mainly accreted onto the massive star because its gravitational capture radius is larger. Also, the "bridge" is significantly tilted, because the flux of the matter that rounds the secondary is higher. As a consequence, a part of the spiral stream moving along the "bridge" toward the primary directly collides with its accretion disk. As a result, the shock wave is formed on the edge of the accretion disk. It leads to an increase in the loss of angular momentum in the primary accretion disk. Thus, despite the higher matter flux from the protoplanetary disk toward the secondary, the rate of accretion onto the primary is higher.

\section{Conclusions}

- Due to the supersonic motion of the components of TTSs, two bow-shocks are formed in the circumbinary disks of these systems. In the inner region of the binary, the velocity distribution is far from the Keplerian one and the flow structure is mainly governed by the bow-shocks.

- Existence of the shocks leads to redistribution of the angular momentum in the protoplanetary disk. As a result, two flows propagating from the inner edge of the protoplanetary disk to the components are formed.

- The flux of matter from the inner edge of the protoplanetary disk toward the less massive component is larger.

- The circumstellar accretion disk cannot accept all the falling matter. This leads to the complex redistribution of matter in the region between the circumstellar accretion disks. As a consequence, despite the higher matter flux from the protoplanetary disk toward the secondary, most of the matter is accreted onto the primary component.

\section{Acknowledgements}

This work was supported by the Basic Research Program of the Presidium of the Russian Academy of Sciences, Russian Foundation for Basic Research (projects 09-02-00064, 09-02-00993, 11-02-00076, 11-02-01248), and the Federal Targeted Program "Science and Science Education for Innovation in Russia 2009-2013."

\section{References}

Artymowicz, P. \& Lubow, S. H. 1994, ApJ, 421, 651

Fateeva, A. M., Bisikalo, D. V., Kaygorodov, P. V., \& Sytov, A. Y. 2011, Ap\& $S S$, 335, 125

Kaigorodov, P. V., Bisikalo, D. V., Fateeva, A. M., \& Sytov, A. Y. 2010, Astron. Rep., 54, 1078

Sytov, A. Y., Kaygorodov, P. V., Fateeva, A. M., \& Bisikalo, D. V. 2011, Astron. Rep., 55, 793 